\title{
The Function of RNAi in Plant Development
}

\author{
R.S. Poethig, A. Peragine, M. Yoshikawa, ${ }^{*}$ C. Hunter, M. Willmann, and G. Wu \\ Department of Biology, University of Pennsylvania, Philadelphia, Pennsylvania 19104-6018
}

\begin{abstract}
The morphological phenotype of mutations in genes required for posttranscriptional gene silencing (PTGS) or RNA interference (RNAi) in Arabidopsis demonstrates that this process is critical for normal development. One way in which RNAi contributes to gene regulation is through its involvement in the biogenesis of trans-acting small interfering RNAs (siRNAs). These endogenous siRNAs are derived from noncoding transcripts that are cleaved by a microRNA (miRNA) and mediate the silencing of protein-coding transcripts. Some protein-coding genes are also subject to miRNA-initiated transitive silencing. Several developmentally important transcription factors regulated by these silencing mechanisms have been identified.
\end{abstract}

Plants and animals produce two major types of 21- to 24-nucleotide RNAs: miRNAs and siRNAs (for review, see Du and Zamore 2005; Carthew 2006; Jones-Rhoades et al. 2006; Mallory and Vaucheret 2006; ValenciaSanchez et al. 2006; Vazquez 2006). miRNAs are endogenously encoded and typically regulate genes involved in development or physiology. In contrast, siRNAs are the by-product of transcriptional or posttranscriptional silencing pathways, and in plants were thought to function primarily to suppress the expression of potentially deleterious RNAs, such as RNA derived from viruses and transposons. However, recent studies have revealed an unexpected role for RNAi in developmental gene regulation in plants and have led to the discovery of a new class of endogenous siRNAs, termed trans-acting siRNAs (ta-siRNAs) (Peragine et al. 2004; Vazquez et al. 2004; Allen et al. 2005; Williams et al. 2005; Yoshikawa et al. 2005). ta-siRNAs are interesting not only because of their regulatory function, but also because their biogenesis is dependent on miRNA-directed cleavage of a precursor transcript (Allen et al. 2005; Yoshikawa et al. 2005). These results not only have expanded our understanding of the normal function of RNAi in plants, but also have established a novel function for miRNAdirected transcript cleavage.

\section{IDENTIFICATION OF TA-SIRNAS}

ta-siRNAs are 21-nucleotide siRNAs that posttranscriptionally repress the expression of transcripts to which they have imperfect complementarity. ta-siRNAs were discovered independently in our laboratory (Peragine et al. 2004) and in the laboratory of $\mathrm{H}$. Vaucheret (Vazquez et al. 2004). We identified this novel class of siRNAs from an analysis of mutations that affect the process of vegetative phase change in Arabidopsis. Like all flowering plants, Arabidopsis undergoes a transition from a juvenile to an adult phase of vegetative devel-

\footnotetext{
*Present address: Department of Molecular Genetics, National Institute of Agrobiological Sciences, Tsukuba, Japan.
}

opment prior to flowering (Brink 1962; Telfer et al. 1997; Kerstetter and Poethig 1998; Baurle and Dean 2006). This transition is known as vegetative phase change, and in Arabidopsis, it is accompanied by changes in leaf shape and size and by the initiation of trichome production on the lower (abaxial) leaf surface (Chien and Sussex 1996; Telfer et al. 1997; Tsukaya et al. 2000). Screens for mutations that accelerate the appearance of adult vegetative traits have produced genes that fall into roughly two phenotypic classes. One class of mutations dramatically accelerates the appearance of adult vegetative traits and essentially eliminates the production of transition leaves. This class includes alleles of the miRNA export receptor HST (Telfer and Poethig 1998; Bollman et al. 2003), the cyclophilin 40 ortholog SQN (Berardini et al. 2001), and the miRNA-processing gene $S E$ (Clarke et al. 1999; Prigge and Wagner 2001; Grigg et al. 2005; Yang et al. 2006). The second class of mutations includes alleles of ZIP/AGO7, RDR6, SGS3, and DCL4. Mutations of these genes have the same morphological phenotype, first described in detail for mutations of ZIP/AGO7 (Fig. 1A,B) (Hunter et al. 2003). In contrast to the first class of mutations, which have a very pleiotropic phenotype, mutations in this second class have specific effects on leaf and flower development. These mutants precociously express a variety of traits associated with the adult phase of vegetative development, including epinastic leaf curvature, abaxial trichome production, and increased hydathode production and cause the septum of the silique to develop stigmatic tissue. Double- or triple-mutant combinations of these genes have a phenotype that is nearly identical to single mutants, implying that they operate in the same pathway (Fig. 1B) (Peragine et al. 2004).

Because two of this latter class of genes, SGS3 and $R D R 6$, had been shown to promote posttranscriptional silencing of sense transgenes in Arabidopsis (Dalmay et al. 2000; Mourrain et al. 2000), we reasoned that their phenotype was likely due to the up-regulation of genes that are normally silenced during the juvenile phase of development. To identify these genes, we performed a microarray analysis of RNA levels in zip, sgs 3 , and $r d r 6$ seedlings (Peragine et al. 2004). This analysis revealed two genes whose transcripts are elevated by $s g s 3$ and $r d r 6$ 


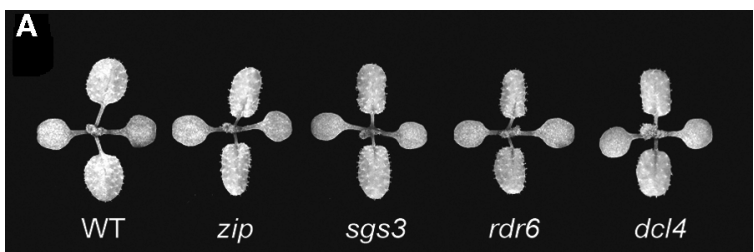

$\mathbf{B}$

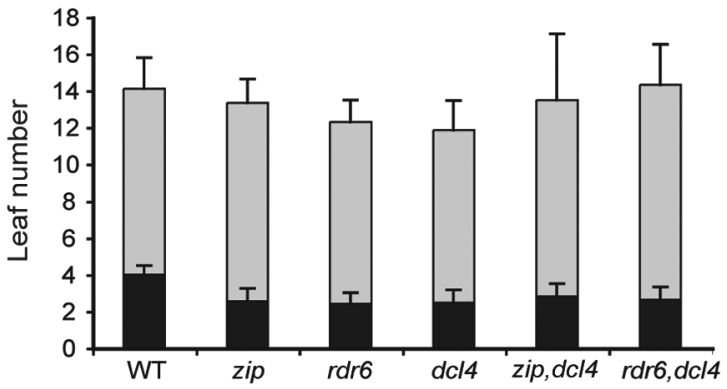

Figure 1. Phenotype of mutations in genes required for the biogenesis of ta-siRNAs in Arabidopsis. (A) 10-day-old seedlings of wild-type (WT), zip-1, sgs3-11, rdr6-11, and $d c l 4-2$. (B) The number of juvenile and adult leaves in wild type and mutant plants ( \pm S.D.). Juvenile leaves are leaves that lack abaxial trichomes. The phenotype of double mutants is nearly identical to that of single mutants. The interaction between zip-1, sgs3-11, and $r d r 6-11$ has been described previously (Peragine et al. 2004).

but not by zip, and three genes that are elevated by all three of these mutations. We were unable to identify siRNAs from these genes among known endogenous siRNAs. However, one up-regulated gene (At5g18040) had near-perfect reverse complementarity to a cloned siRNA (siR255/siR480) from an intergenic region of the genome. RNA ligase-mediated rapid amplification of 5' cDNA ends (RLM-5' RACE) revealed that At5g18040 is cleaved in the middle of this complementary region, supporting the conclusion that it is negatively regulated by siR255/siR480. sgs 3 and $r d r 6$ were found to block the production of $\operatorname{siR} 255 / \mathrm{siR} 480$, accounting for the elevation of At5g18040 in these mutants. Similar results for these genes were obtained by Vazquez et al. (2004). Subsequent studies by us (Yoshikawa et al. 2005) and other workers (Allen et al. 2005; Williams et al. 2005) revealed that most of the other up-regulated genes identified in this microarray analysis are also targets of siRNAs from unrelated transcripts and showed that the elevated expression of these genes in zip, $r d r 6$, and $\operatorname{sgs} 3$ is correlated with the absence of these "trans-acting" siRNAs.

Five loci capable of producing ta-siRNAs-TAS1a, $T A S 1 b, T A S 1 c, T A S 2$, and TAS3- have been identified in Arabidopsis (Fig. 2). TAS1 $a, b$, and $c$ are closely related and produce ta-siRNAs that target At5g18040 and members of a large family of closely related genes encoding pentatricopeptide repeat (PPR) proteins (Peragine et al. 2004; Vazquez et al. 2004; Yoshikawa et al. 2005). TAS2 is a paralog of the TAS1 loci, but it only produces tasiRNAs that target PPR genes (Allen et al. 2005;

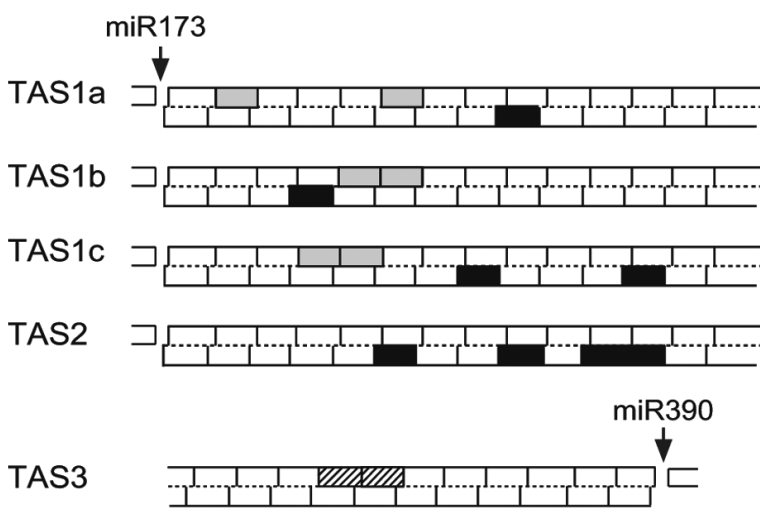

Figure 2. Sources of ta-siRNAs in Arabidopsis. Most of the siRNAs from these loci are in phase with a miRNA cleavage site. Only confirmed or predicted ta-siRNAs are illustrated. Targets of ta-siRNAs: (black) PPR genes; (gray) At5g18040-related genes; (diagonal lines) ARF2, ETT/ARF3, and ARF4.

Yoshikawa et al. 2005). TAS1 and TAS2 do not appear to be conserved outside Arabidopsis thaliana. TAS3 regulates three members of the ARF family of auxin-related transcription factors ( $A R F 2, E T T / A R F 3$, and $A R F 4)$ and is conserved throughout higher plants (Allen et al. 2005; Williams et al. 2005).

\section{BIOGENESIS OF TA-SIRNAS}

How are ta-siRNAs generated? Vazquez et al. (2004) reported that most of the 21-nucleotide siRNAs from TASI $a$ are in exact register with each other. The basis for this pattern became apparent when it was discovered that siRNAs from TAS1, TAS2, and TAS3 are all in phase with a miRNA cleavage site. TAS1 and TAS2 are targets of $m i R 173$, and TAS3 is a target of miR390 (Allen et al. 2005; Yoshikawa et al. 2005). This immediately suggested that ta-siRNAs are derived from progressive, Dicer-mediated cleavage, starting at the miRNA target site. Evidence that miRNA-directed cleavage is indeed essential for the biogenesis of ta-siRNAs was provided by the reconstitution of TASIa processing in a heterologous system (Allen et al. 2005) and by the observation that tasiRNA biogenesis is blocked by mutations in miR173 (Gasciolli et al. 2005) or mutations that disrupt miRNA biogenesis (Yoshikawa et al. 2005).

The specific function of RDR6, SGS3, and DCL4 in the biogenesis of ta-siRNAs was determined from an analysis of the effect of mutations in these genes on the primary transcripts from TAS1a and TAS2 (Fig. 3) (Yoshikawa et al. 2005). $R D R 6$ and $S G S 3$ were originally identified in screens for mutants that block the posttranscriptional silencing of transgenes (Dalmay et al. 2000; Mourrain et al. 2000). RDR6 was proposed to be responsible for the transformation of mRNA into double-stranded RNA based on its similarity to a tomato RNA-dependent RNA polymerase and the observation that it is required for the silencing of transgenes that produce "sense" transcripts, but not transgenes that have been engineered to produce a hairpin transcript (Beclin et al. 2002). Additional evi- 


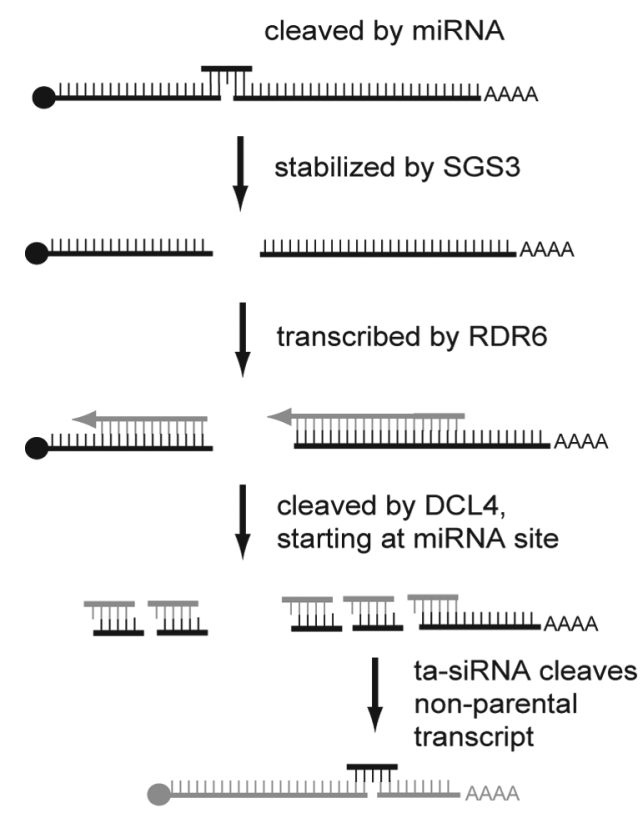

Figure 3. Pathway for the biogenesis of ta-siRNAs.

dence for this conclusion was provided by the observation that RDR6 is required for transitivity - the production of siRNAs from an untargeted region of a transcript (Vaistij et al. 2002). SGS3 is a plant-specific protein of unknown function. Although mutations in these genes have identical effects on transgene silencing (Beclin et al. 2002) and have identical morphological phenotypes (Peragine et al. 2004), they have opposite effects on the accumulation of the miR173-generated cleavage fragments of TAS2 and TASla (Yoshikawa et al. 2005). sgs 3 decreases the accumulation of these fragments, and $r d r 6$ produces a dramatic increase in their accumulation. sgs 3 rdr6 double mutants have the same phenotype as $s g s 3$, indicating that $S G S 3$ is required for the accumulation of these fragments both in the presence and in the absence of RDR6 function. This epistatic interaction suggests that SGS3 somehow stabilizes these fragments before they are transcribed by RDR6. The subsequent cleavage of double-stranded transcripts into 21-nucleotide siRNAs is accomplished by DCL4, one of four Dicer-like proteins in Arabidopsis (Dunoyer et al. 2005; Gasciolli et al. 2005; Xie et al. 2005; Yoshikawa et al. 2005). The R2D2-like protein, DRB4, binds to DCL4 (Hiraguri et al. 2005) and may assist in its activity because the phenotype of a $d r b 4$ mutation resembles dcl4 (Adenot et al. 2006). TAS2 and TAS3 are also processed inefficiently by DCL2 into 22nucleotide siRNAs and by DCL3 into 24-nucleotide siRNAs (Gasciolli et al. 2005; Bouche et al. 2006; Henderson et al. 2006).

\section{FUNCTION OF TA-SIRNAS}

The loss-of-function phenotype of genes required for RNAi in Arabidopsis demonstrates that this process has a role in leaf and flower development, although the subtle nature of their phenotype demonstrates that this role is relatively limited. Most of the up-regulated genes identified by transcript profiling of zip, $r d r 6$, and $s g s 3$ are targets of ta-siRNAs, implying that defects in this class of siRNAs largely account for the mutant phenotype of these genes. T-DNA (transferred DNA) insertions in TAS1 and TAS2 have no apparent morphological phenotype (Vazquez et al. 2004; Yoshikawa et al. 2005). In contrast, a T-DNA insertion in TAS3 has a phenotype quite similar to that of zip, rdr6, sgs3, and dcl4 (Adenot et al. 2006). $T A S 3$ produces a ta-siRNA (tasi-ARF) that targets members of the ARF family of auxin-related transcription factors (Allen et al. 2005; Williams et al. 2005). Two of the targets of tasi-ARF-ETT/ARF3 and ARF4 - are elevated in zip, sgs $3, r d r 6$, and $d c l 4$, and the precocious phenotype of these mutations is mimicked by transgenes that express a tasi-ARF-insensitive form of ETT/ARF3 (Fahlgren et al. 2006; Hunter et al. 2006). Additional evidence that $A R F 3$ and $A R F 4$ are responsible for the phenotype of zip, $r d r 6$, $s g s 3$, and $d c l 4$ was provided by the isolation of loss-offunction mutations of these genes in a screen for secondsite suppressors of zip (Hunter et al. 2006). We found that ett and arf4 partially suppress the phenotype of zip and $r d r 6$, demonstrating that the wild-type alleles of these genes are necessary for the zip mutant phenotype. The observation that neither single mutant is capable of completely suppressing the phenotype of zip suggests that both genes contribute to this phenotype, and that they have similar, but not identical, functions in leaf development. This conclusion is also supported by the observation that the level of $A R F 3$ mRNA necessary to replicate the phenotype of zip (in plants transformed with $35 S:: A R F 3)$ is considerably higher than the level of $A R F 3$ mRNA in zip mutants (Hunter et al. 2006).

The loss-of-function phenotypes of ETT/ARF3 and $A R F 4$ demonstrate that these genes have several functions in leaf development. Mutations in ETT/ARF3 were originally identified because of their effects on carpel polarity (Sessions and Zambryski 1995; Sessions et al. 1997). Subsequent studies showed that ETT/ARF3 and ARF4 regulate leaf polarity as well, probably in conjunction with the $K A N$ family of transcription factors (Pekker et al. 2005; Hunter et al. 2006). Strong ett/arf3 mutations simplify the shape of spongy mesophyll cells in a manner similar to that of kan1, and ett/arf3 arf4 double mutants resemble kan1 kan2 double mutants. Furthermore, ett/arf 3 is able to suppress the floral phenotype of KAN1 overexpression (Pekker et al. 2005). Because many of the traits that change during vegetative development are polarized (e.g., trichome production and leaf curvature), it may be that temporal changes in the regulation of leaf polarity are the basis for the phase-specific expression pattern of these traits. It is unlikely that this is the sole function of $E T T / A R F 3$ and $A R F 4$, however, because some aspects of their mutant phenotype cannot be readily explained by a loss of abaxial identity. In particular, both mutations delay abaxial trichome production and affect the length:width ratio of the lamina (Hunter et al. 2006). Because defects in the specification of abaxial identity typically result in enhanced abaxial trichome production, the trichome phenoytpe of ett/arf3 and arf4 is inconsistent with a defect in leaf polarity. 


\section{IS RNAI of MIRNA-REGULATED GENES FUNCTIONALLY IMPORTANT?}

The fact that transcript profiling of zip, sgs3, and $r d r 6$ produced only a small number of up-regulated genes suggests that RNAi does not make a major contribution to the regulation of gene expression in Arabidopsis (Peragine et al. 2004). However, northern analysis of several miRNAregulated protein-coding genes has revealed that these genes are the source of siRNAs and that these siRNAs are dependent on AGO1, DCL1, DCL3, RDR6, and RDR2 (Ronemus et al. 2006). Thus, at least some miRNA-regulated protein-coding genes appear to be subject to RNAi, presumably as a result of being cleaved by a miRNA. Whether this process has a significant role in the expression of these genes remains to be determined.

One miRNA-regulated gene whose expression is elevated in zip, rdr6, and sgs 3 is SPL3. SPL3 encodes a member of the Squamosa promoter-binding family of transcription factors (Cardon et al. 1999), ten of which possess a cognate site for miR156 (Rhoades et al. 2002). SPL3 is particularly interesting because its mRNA increases early in vegetative development, coincident with a decrease in the level of miR156 (Wu and Poethig 2006). This temporal change in SPL3 expression is also observed with a GUS-SPL3 fusion protein under the transcriptional regulation of the cauliflower mosaic virus (CaMV) 35S promoter and is dependent on the presence of a functional miR156 target site, indicating that it is likely a consequence of the decrease in miR156. Misexpression of SPL3, or its close relatives SPL4 and SPL5, as a consequence of the loss of their miR156 target site results in precocious vegetative phase change and early flowering. These results suggest that these genes have a critical role in vegetative phase change and flowering and raise the possibility that changes in the expression of miR156 may have an important role in these transitions (Wu and Poethig 2006).

What is the function of ZIP, SGS3, and RDR6 in the regulation of SPL3? The observation that SPL3 is up-regulated in these mutants indicates that it is either directly or indirectly subject to RNAi. One possibility is that cleavage of SPL3 by miR 156 makes it susceptible to transitive silencing, just as miRNA-directed cleavage initiates the production of siRNAs from the precursors of ta-siRNAs (Allen et al. 2005; Yoshikawa et al. 2005). If transitive silencing had a critical role in the activity of miR156, one might expect mutations in this process to have a phenotype similar to that of mutations that affect the biogenesis of miR156. However, zip and rdr6 only cause a slight, temporally uniform increase in the abundance of SPL3 mRNA, whereas $h s t$ - a mutation that dramatically reduces $m i R 156$ levels - produces a very large and temporally variable increase in SPL3 (Wu and Poethig 2006). Furthermore, we were unable to detect siRNAs derived from SPL3 by northern analysis and did not find any evidence for the existence of these siRNAs in databases of endogenous small RNAs in Arabidopsis. Although the possibility that these siRNAs may be present at extremely low levels has not been eliminated, the existing evidence suggests that the effect of zip, sgs3, and $r d r 6$ on SPL3 expression may be an indirect effect of these mutations on a transcriptional regulator of SPL3.

\section{FUNCTION OF RNAI IN VEGETATIVE PHASE CHANGE}

The results described above demonstrate that RNAi normally promotes the expression of juvenile traits by repressing the expression of genes such as ETT/ARF3, $A R F 4$, and SPL3 during this phase. Therefore, one way in which phase change might be regulated is by a decrease in the activity of this silencing pathway late in shoot development. There is currently no support for this hypothesis, however. We observed no change in the abundance of tasi-ARF during development, nor did we detect a difference in the expression of its targets $E T T / A R F 3$ and $A R F 4$ in successive leaves (Hunter et al. 2006). Similarly, zip and $r d r 6$ cause a uniform increase in the expression of SPL3, rather than affecting the temporal expression pattern of this gene (Wu and Poethig 2006). These data are consistent with the observation that $s g s 3$ and $r d r 6$ block transgene silencing throughout shoot development (Dalmay et al. 2000; Mourrain et al. 2000; Peragine et al. 2004) and suggest that there is no major temporal change in the activity of the posttranscriptional gene silencing pathway during shoot growth. We conclude from these observations that RNAi regulates the sensitivity of the shoot apex to factors that regulate phase change, rather than being a component of the phase change signal.

Although there is no evidence that temporal changes in the expression of ZIP, SGS3, RDR6, and DCL4 contribute to vegetative phase change, this may not be true for components of the transcriptional silencing pathway. Temporal variation in gene silencing has been described for transposons (Banks et al. 1988; Fedoroff and Banks 1988; Martienssen et al. 1990) and the $\mathrm{Pl}-\mathrm{Bh}$ mutation (Cocciolone and Cone 1993; Hoekenga et al. 2000; Irish and McMurray 2006) in maize, as well as for transgenes in tobacco (de Carvalho et al. 1992) and Arabidopsis (Elmayan et al. 1998; Vaucheret et al. 2004). In general, silencing increases along the length of the shoot; genes subject to silencing are typically expressed at a higher level in organs produced early in development (cotyledons, juvenile leaves) than in organs produced later in shoot development (adult leaves, flowers). In maize, this temporal decrease in gene expression or transposon activity has been associated with increased DNA methylation (Banks et al. 1988; Fedoroff and Banks 1988; Martienssen et al. 1990; Hoekenga et al. 2000; Irish and McMurray 2006), and there is evidence that DNA methylation also increases during shoot development in Arabidopsis (RuizGarcia et al. 2005). Whether this temporal change in gene silencing and DNA methylation represents the accumulation of changes that occur at a constant rate during shoot growth or is the result of a discrete change in the activity of a gene silencing pathway is unknown.

\section{CONCLUSIONS}

tasi-ARF and its targets are present in many flowering plants and in at least one gymnosperm (Allen et al. 2005; 
Williams et al. 2005). Furthermore, miR390 - the miRNA that is responsible for the production of tasi-ARF - is conserved in moss (Axtell and Bartel 2005). Although the targets of miR390 in moss have not yet been identified, these observations indicate that ta-siRNAs appeared early in plant evolution. Given that ta-siRNAs are ancient and effective mechanisms for simultaneously regulating a large number of related and unrelated genes, one might imagine that this mechanism would have been strongly selected in the evolution of regulatory pathways. However, there is little evidence for this conclusion. Mutations that interfere with RNAi have very subtle phenotypes in Arabidopsis, and only a few of the known miRNA targets in Arabidopsis are affected by these mutations. Although additional sources of ta-siRNAs probably remain to be discovered, current evidence suggests that the number of such loci in Arabidopsis is not large. Why are ta-siRNAs in particular, and RNAi in general, not more widely used in developmental regulation? Did this process evolve for a purpose (e.g., virus resistance) that makes it difficult to use it for alternative purposes? This might be the case, for example, if the viral RNA substrates of RNAi and endogenous mRNAs are structurally different in some way or are shunted to different processing pathways. Or, more interestingly, does RNAi have properties that make it more useful for regulating some types of processes than others? Answers to these questions will have to await a better understanding of the mechanism of RNAi in plants and the factors that make transcripts susceptible to this process.

\section{ACKNOWLEDGMENTS}

We thank members of the Poethig lab for helpful discussions. This research was supported by a National Institutes of Health training grant to A.P., NIH postdoctoral fellowships to M.W. and C.H., and an NIH research grant to R.S.P.

\section{REFERENCES}

Adenot X., Elmayan T., Lauressergues D., Boutet S., Bouche N., Gasciolli V., and Vaucheret H. 2006. DRB4-dependent TAS3 trans-acting siRNAs control leaf morphology through AGO7. Curr. Biol. 16: 927.

Allen E., Xie Z., Gustafson A.M., and Carrington J.C. 2005. microRNA-directed phasing during trans-acting siRNA biogenesis in plants. Cell 121: 207.

Axtell M.J. and Bartel D.P. 2005. Antiquity of microRNAs and their targets in land plants. Plant Cell 17: 1658.

Banks J.A., Masson P., and Fedoroff N. 1988. Molecular mechanisms in the developmental regulation of the maize Suppressor-mutator transposable element. Genes Dev. 2: 1364 .

Baurle I. and Dean C. 2006. The timing of developmental transitions in plants. Cell 125: 655.

Beclin C., Boutet S., Waterhouse P., and Vaucheret H. 2002. A branched pathway for transgene-induced RNA silencing in plants. Curr. Biol. 12: 684.

Berardini T.Z., Bollman K., Sun H., and Poethig R.S. 2001. Regulation of vegetative phase change in Arabidopsis thaliana by cyclophilin 40. Science 291: 2405.

Bollman K.M., Aukerman M.J., Park M.Y., Hunter C., Berardini T.Z., and Poethig R.S. 2003. HASTY, the Arabidopsis ortholog of Exportin 5/MSN5, regulates phase change and morphogenesis. Development 130: 1493.

Bouche N., Lauressergues D., Gasciolli V., and Vaucheret H. 2006. An antagonistic function for Arabidopsis DCL2 in development and a new function for DCL4 in generating viral siRNAs. EMBO J. 25: 3347.

Brink R.A. 1962. Phase change in higher plants and somatic cell heredity. Q. Rev. Biol. 37: 1.

Cardon G., Hohmann S., Klein J., Nettesheim K., Saedler H., and Huijser P. 1999. Molecular characterisation of the Arabidopsis SBP-box genes. Gene 237: 91.

Carthew R.W. 2006. Gene regulation by microRNAs. Curr. Opin. Genet. Dev. 16: 203.

Chien J.C. and Sussex I.M. 1996. Differential regulation of trichome formation on the adaxial and abaxial leaf surfaces by gibberellins and photoperiod in Arabidopsis thaliana (L) Heynh. Plant Physiol. 111: 1321.

Clarke J.H., Tack D., Findlay K., Van Montagu M., and Van Lijsebettens M. 1999. The SERRATE locus controls the formation of the early juvenile leaves and phase length in Arabidopsis. Plant J. 20: 493.

Cocciolone S.M. and Cone K.C. 1993. Pl-Bh, an anthocyanin regulatory gene of maize that leads to variegated pigmentation. Genetics 135: 575.

Dalmay T., Hamilton A., Rudd S., Angell S., and Baulcombe D.C. 2000. An RNA-dependent RNA polymerase gene in Arabidopsis is required for posttranscriptional gene silencing mediated by a transgene but not by a virus. Cell 101: 543 .

de Carvalho F., Gheysen G., Kushnir S., Van Montagu M., Inze D., and Castresana C. 1992. Suppression of beta-1,3-glucanase transgene expression in homozygous plants. EMBO J. 11: 2595 .

Du T. and Zamore P.D. 2005. microPrimer: The biogenesis and function of microRNA. Development 132: 4645.

Dunoyer P., Himber C., and Voinnet O. 2005. DICER-LIKE 4 is required for RNA interference and produces the 21-nucleotide small interfering RNA component of the plant cell-to-cell silencing signal. Nat. Genet. 37: 1356.

Elmayan T., Balzergue S., Beon F., Bourdon V., Daubremet J., Guenet Y., Mourrain P., Palauqui J.C., Vernhettes S., Vialle T., et al. 1998. Arabidopsis mutants impaired in cosuppression. Plant Cell 10: 1747.

Fahlgren N., Montgomery T.A., Howell M.D., Allen E., Dvorak S.K., Alexander A.L., and Carrington J.C. 2006. Regulation of AUXIN RESPONSE FACTOR3 by TAS3 ta-siRNA affects developmental timing and patterning in Arabidopsis. Curr. Biol. 16: 939.

Fedoroff N.V. and Banks J.A. 1988. Is the Suppressor-mutator element controlled by a basic developmental regulatory mechanism? Genetics 120: 559.

Gasciolli V., Mallory A.C., Bartel D.P., and Vaucheret H. 2005. Partially redundant functions of Arabidopsis DICER-like enzymes and a role for DCL4 in producing trans-acting siRNAs. Curr. Biol. 15: 1494.

Grigg S.P., Canales C., Hay A., and Tsiantis M. 2005. SERRATE coordinates shoot meristem function and leaf axial patterning in Arabidopsis. Nature 437: 1022.

Henderson I.R., Zhang X., Lu C., Johnson L., Meyers B.C., Green P.J. and Jacobsen S.E. 2006. Dissecting Arabidopsis thaliana DICER function in small RNA processing, gene silencing and DNA methylation patterning. Nat. Genet. 38: 721.

Hiraguri A., Itoh R., Kondo N., Nomura Y., Aizawa D., Murai Y., Koiwa H., Seki M., Shinozaki K., and Fukuhara T. 2005. Specific interactions between Dicer-like proteins and HYL1/DRB-family dsRNA-binding proteins in Arabidopsis thaliana. Plant Mol. Biol. 57: 173.

Hoekenga O.A., Muszynski M.G. and Cone K.C. 2000. Developmental patterns of chromatin structure and DNA methylation responsible for epigenetic expression of a maize regulatory gene. Genetics 155: 1889 .

Hunter C., Sun H., and Poethig R.S. 2003. The Arabidopsis heterochronic gene ZIPPY is an ARGONAUTE family member. Curr. Biol. 13: 1734. 
Hunter C., Willmann M.R., Wu G., Yoshikawa M., de la Luz Gutierrez-Nava M., and Poethig S.R. 2006. Trans-acting siRNA-mediated repression of ETTIN and ARF4 regulates heteroblasty in Arabidopsis. Development 133: 2973.

Irish E.E. and McMurray D. 2006. Rejuvenation by shoot apex culture recapitulates the developmental increase of methylation at the maize gene Pl-Blotched. Plant Mol. Biol. 60: 747.

Jones-Rhoades M.W., Bartel D.P., and Bartel B. 2006. MicroRNAs and their regulatory roles in plants. Annu. Rev. Plant Biol. 57: 19.

Kerstetter R.A. and Poethig R.S. 1998. The specification of leaf identity during shoot development. Annu. Rev. Cell Dev. Biol. 14: 373

Mallory A.C. and Vaucheret H. 2006. Functions of microRNAs and related small RNAs in plants. Nat. Genet. (suppl.) 38: S31.

Martienssen R., Barkan A., Taylor W.C., and Freeling M. 1990. Somatically heritable switches in the DNA modification of $\mathrm{Mu}$ transposable elements monitored with a suppressible mutant in maize. Genes Dev. 4: 331.

Mourrain P., Beclin C., Elmayan T., Feuerbach F., Godon C., Morel J.B., Jouette D., Lacombe A.M., Nikic S., Picault N., et al. 2000. Arabidopsis $S G S 2$ and $S G S 3$ genes are required for posttranscriptional gene silencing and natural virus resistance. Cell 101: 533.

Pekker I., Alvarez J.P., and Eshed Y. 2005. Auxin response factors mediate Arabidopsis organ asymmetry via modulation of KANADI activity. Plant Cell 17: 2899.

Peragine A., Yoshikawa M., Wu G., Albrecht H.L., and Poethig R.S. 2004. $S G S 3$ and $S G S 2 / S D E 1 / R D R 6$ are required for juvenile development and the production of trans-acting siRNAs in Arabidopsis. Genes Dev. 18: 2368.

Prigge M.J. and Wagner D.R. 2001. The Arabidopsis SERRATE gene encodes a zinc-finger protein required for normal shoot development. Plant Cell 13: 1263.

Rhoades M.W., Reinhart B.J., Lim L.P., Burge C.B., Bartel B., and Bartel D.P. 2002. Prediction of plant microRNA targets. Cell 110: 513.

Ronemus M., Vaughn M.W., and Martienssen R. A. 2006. MicroRNA-targeted and small interfering RNA-mediated mRNA degradation is regulated by ARGONAUTE, DICER, and RNA-dependent RNA polymerase in Arabidopsis. Plant Cell 18: 1559.

Ruiz-Garcia L., Cervera M.T., and Martinez-Zapater J.M. 2005. DNA methylation increases throughout Arabidopsis development. Planta 222: 301.

Sessions R.A. and Zambryski P.C. 1995. Arabidopsis gynoecium structure in the wild and in ettin mutants. Development 121: 1519 .
Sessions A., Nemhauser J.L., McColl A., Roe J.L., Feldmann K.A., and Zambryski P.C. 1997. ETTIN patterns the Arabidopsis floral meristem and reproductive organs. Development 124: 4481.

Telfer A. and Poethig R.S. 1998. HASTY: A gene that regulates the timing of shoot maturation in Arabidopsis thaliana. Development 125: 1889.

Telfer A., Bollman K.M., and Poethig R.S. 1997. Phase change and the regulation of trichome distribution in Arabidopsis thaliana. Development 124: 645.

Tsukaya H., Shoda K., Kim G.T., and Uchimiya H. 2000. Heteroblasty in Arabidopsis thaliana (L.) Heynh. Planta 210: 536.

Vaistij F.E., Jones L., and Baulcombe D.C. 2002. Spreading of RNA targeting and DNA methylation in RNA silencing requires transcription of the target gene and a putative RNAdependent RNA polymerase. Plant Cell 14: 857.

Valencia-Sanchez M.A., Liu J., Hannon G.J., and Parker R. 2006. Control of translation and mRNA degradation by miRNAs and siRNAs. Genes Dev. 20: 515.

Vaucheret H., Vazquez F., Crete P., and Bartel D.P. 2004. The action of ARGONAUTE1 in the miRNA pathway and its regulation by the miRNA pathway are crucial for plant development. Genes Dev. 18: 1187.

Vazquez F. 2006. Arabidopsis endogenous small RNAs: Highways and byways. Trends Plant Sci. 11: 460.

Vazquez F., Vaucheret H., Rajagopalan R., Lepers C., Gasciolli V., Mallory A.C., Hilbert J.L., Bartel D.P., and Crete P. 2004. Endogenous trans-acting siRNAs regulate the accumulation of Arabidopsis mRNAs. Mol. Cell 16: 69.

Williams L., Carles C.C., Osmont K.S., and Fletcher J.C. 2005. A database analysis method identifies an endogenous transacting short-interfering RNA that targets the Arabidopsis $A R F 2, A R F 3$, and ARF4 genes. Proc. Natl. Acad. Sci. 102: 9703.

Wu G. and Poethig R. S. 2006. Temporal regulation of shoot development in Arabidopsis thaliana by miR156 and its target SPL3. Development 133: 3539.

Xie Z., Allen E., Wilken A., and Carrington J.C. 2005. DICERLIKE 4 functions in trans-acting small interfering RNA biogenesis and vegetative phase change in Arabidopsis thaliana. Proc. Natl. Acad. Sci. 102: 12984.

Yang L., Liu Z., Lu F., Dong A., and Huang H. 2006. SERRATE is a novel nuclear regulator in primary microRNA processing in Arabidopsis. Plant J. 47: 841.

Yoshikawa M., Peragine A., Park M.Y., and Poethig R.S. 2005. A pathway for the biogenesis of trans-acting siRNAs in Arabidopsis. Genes Dev. 19: 2164. 


\section{$8_{\mathrm{CSH}}^{\infty} \mathrm{H}$ Cold Spring Harbor Symposia SYMPOSIA}

\section{The Function of RNAi in Plant Development}

R.S. POETHIG, A. PERAGINE, M. YOSHIKAWA, et al.

Cold Spring Harb Symp Quant Biol 2006 71: 165-170

Access the most recent version at doi:10.1101/sqb.2006.71.030

References This article cites 56 articles, 27 of which can be accessed free at: http://symposium.cshlp.org/content/71/165.full.html\#ref-list-1

\section{License}

Email Alerting Receive free email alerts when new articles cite this article - sign up in Service the box at the top right corner of the article or click here. 